\title{
HOLOMORPHIC CUBIC DIFFERENTIALS AND MINIMAL LAGRANGIAN SURFACES IN $\mathbb{C H}^{2}$
}

\author{
Zheng Huang, John Loftin And Marcello Lucia
}

\begin{abstract}
Minimal Lagrangian submanifolds of a Kähler manifold represent a very interesting class of submanifolds as they are Lagrangian with respect to the symplectic structure of the ambient space, while minimal with respect to the Riemannian structure. In this paper, we study minimal Lagrangian immersions of the universal cover of closed surfaces (of genus $g \geq 2$ ) in $\mathbb{C H}^{2}$, with prescribed data $(\sigma, t q$ ), where $\sigma$ is a conformal structure on the surface $S$, and $q d z^{3}$ is a holomorphic cubic differential on the Riemann surface $(S, \sigma)$. We show existence and non-uniqueness of such minimal Lagrangian immersions. We analyze the asymptotic behaviors for such immersions, and establish the surface area with respect to the induced metric as a Weil-Petersson potential function for the space of holomorphic cubic differentials on $(S, \sigma)$.
\end{abstract}

\section{Introduction}

The theory of minimal hypersurfaces (co-dimension one) in a Riemannian manifold has been a field of both extraordinary depth and far-reaching width in mathematics. The situation of higher co-dimensional minimal submanifolds can be much more complicated. In this paper, we aim to investigate some minimal submanifolds of codimension two, motivated from mirror symmetry and "Lagrangian Plateau problem" [SW99, SW01]. The minimal Lagrangian submanifolds in various ambient spaces can be studied as a constrained variational problem (see for instance [Oh90, MW93]), and there are many interesting analogs to the classical minimal surface theory in Riemannian manifolds. In general, there are obstructions to the existence of minimal Lagrangian submanifolds in a Riemannian manifold, even in the case of a Kähler manifold [Bry87].

For a Kähler manifold $M^{2 n}$, one studies its minimal Lagrangian submanifolds: Lagrangian with respect to the symplectic structure and minimal with respect to the Riemannian structure of $M^{2 n}$. The obstructions in [Bry87] for existence do not occur if $M^{2 n}$ is a Kähler-Einstein manifold, but the general existence is still largely unknown. In this paper we consider the existence and multiplicity of minimal Lagrangian immersions of the universal cover of closed Riemann surfaces into the complex hyperbolic plane $\mathbb{C H}^{2}$, with prescribed data on the closed surface. Each such immersion is equivariant with respect to an induced representation of the fundamental group of the surface into $S U(2,1)$.

It is well known that (for instance, [LJ70]), the second fundamental form of a minimal surface in a three-dimensional space form is described as the real part of a holomorphic quadratic differential. An analogous fact is true for minimal Lagrangian

Received by the editors January 27, 2012.

2000 Mathematics Subject Classification. Primary 53C42, Secondary 35J61, 53D12. 
surfaces in $\mathbb{C H}^{2}$, namely, such an immersion can be constructed from a conformal structure on a closed surface and a holomorphic cubic differential on this conformal structure. The space of holomorphic cubic differentials on closed Riemann surfaces is deeply related to the space of convex flat projective structures on the surface [Lof01, Lab07].

Our perspective is to develop a moduli theory for minimal Lagrangian immersions of (the covering spaces of) closed surfaces into $\mathbb{C H}^{2}$. We are particularly interested in the general existence and uniqueness properties of these immersions for prescribed conformal structure and holomorphic cubic differential. Our method of study relies on reducing the immersion problem to the solvability of the following equation from [LM13]:

$$
\Delta u+2-2 e^{u}-16 t^{2}\|q\|^{2} e^{-2 u}=0,
$$

on a compact Riemann surface equipped with a background hyperbolic metric $g_{\sigma}$, holomorphic cubic differential $q$, and real positive parameter $t$. This equation is the integrability condition of a minimal Lagrangian surface in $\mathbb{C H}^{2}$ with induced metric $e^{u} g_{\sigma}$ and the second fundamental form determined by $t q$.

More specifically, given the pair $(\sigma, t q)$, a solution to equation (1.1) gives rise to a Legendrian frame (see Section 2.2) from the universal cover $\tilde{\Sigma}$ to $S U(2,1)$, for a minimal Lagrangian immersion $\varphi$ from $\tilde{\Sigma}$ to $\mathbb{C H}^{2}$. Note that the group of interest $S U(2,1)$ is the triple covering of $P U(2,1)$, the holomorphic isometry group of $\mathbb{C H}^{2}$. Hence, we obtain a natural representation of the fundamental group of $\Sigma$ into $S U(2,1)$ for which the minimal Lagrangian immersion $\varphi$ is equivariant. The perspective of surface group representation theory is explored in more detail in the paper [LM13].

Note that the induced metric provides a conformal structure and a background metric of constant curvature via the uniformization theorem. Since the deformation of the conformal structures on a closed surface is described by Teichmüller theory, we find extra tools for this problem, as well as applications to Teichmüller theory.

Let us fix some notation and basic assumptions that will be frequently used throughout the paper.

(i) Let $\Sigma$ be a smooth, closed, oriented surface of genus $g \geq 2$, and $\sigma$ be a conformal structure on $\Sigma$, with local conformal coordinate $z$. Note that $\sigma$ is a point on Teichmüller space $\mathcal{T}_{g}$ of Riemann surfaces (here we do not distinguish Teichmüller space and moduli space).

(ii) Let $g_{\sigma} d z d \bar{z}$ be the hyperbolic metric (of constant curvature -1 ) on $(\Sigma, \sigma)$, and $\Delta$ is the Laplace operator for $g_{\sigma}$.

(iii) Let $C(\sigma)$ be the space of holomorphic cubic differentials of the form $q(z) d z^{3}$ on $(\Sigma, \sigma)$, where $\frac{\partial q(z)}{\partial \bar{z}}=0$. Note that by the Riemann-Roch theorem, the complex dimension of $C(\sigma)$ is $5 g-5$ (see for example [FK80]).

(iv) We assign the following notation for a holomorphic cubic differential $q(z) d z^{3}$ with respect to the hyperbolic metric $g_{\sigma}$ :

$$
\|q\|=\frac{|q|}{g_{\sigma}^{3 / 2}}
$$


Therefore it generates a natural $L^{2}$-pairing (the Weil-Petersson pairing) of holomorphic cubic differentials in $C(\sigma)$. We always assume $q \not \equiv 0$, but keep in mind that the cubic differential $q$ must necessarily have (finitely many) zeros on $\Sigma$.

We are interested in understanding minimal Lagrangian surfaces in $\mathbb{C H}^{2}$, in particular, the existence, uniqueness and asymptotic behaviors of such immersions. Our main result can be summarized into the following:

Theorem 1.1. Let $\Sigma$ be a closed marked surface of genus $g \geq 2, \sigma \in \mathcal{T}_{g}$ be a conformal structure on $\Sigma$, and $q d z^{3} \in C(\sigma)$ be a holomorphic cubic differential on this marked surface $(\Sigma, \sigma)$, then we have the following:

(i) There is a $T_{0}=T_{0}(\sigma, q)>0$ so that for any $t \in\left(0, T_{0}\right)$, there are at least two immersed minimal Lagrangian immersions from $\tilde{\Sigma}$, the universal cover of $\Sigma$, into $\mathbb{C H}^{2}$, determined by $(\sigma, t q)$;

(ii) There is a minimal Lagrangian immersion from $\tilde{\Sigma}$ into $\mathbb{C H}^{2}$ determined by $\left(\sigma, T_{0} q\right)$

(iii) [LM13] There exists a $T=T(\sigma, q)>0$ such that for any $t>T$, there is no minimal Lagrangian immersion of $\tilde{\Sigma}$ into $\mathbb{C H}^{2}$ determined by $(\sigma, t q)$.

The most technical parts of Theorem 1.1 are the parts (i) and (ii). Our approach for part (i) consists of two steps: we first (see Theorem 3.3) deploy the continuity method to produce a solution curve to equation (1.1), and show corresponding solutions are stable, then we produce an additional solution by the mountain pass theorem for each stable solution obtained in Theorem 3.3. Part (ii) essentially determines the asymptotic behavior of the solution curve on which the linearized operator is positive. Proving part (ii) requires the closedness estimate in Theorem 3.7, i.e., the continuity method extends to the endpoint $T_{0}$. This estimate relies on the compactness of the surface $\Sigma$. We also note an antecedent to part (i) is proved already in [LM13]: there is a $\tilde{T}_{0} \in\left(0, T_{0}\right]$ so that there is a single solution of $(1.1)$ for each $t<\tilde{T}_{0}$ is Theorem 5.1 of [LM13].

As an application to Teichmüller theory, we show that

Theorem 1.2. The induced surface area (for a unique minimal Lagrangian immersion corresponding to data $\left.\left(\sigma, q d z^{3}\right)\right)$ is a potential function of the Weil-Petersson norm in the space of holomorphic cubic differentials on $\sigma \in \mathcal{T}_{g}$.

Understanding Lagrangian surfaces in $\mathbb{C H}^{2}$ is an important ingredient in studying representations in the complex hyperbolic quasi-Fuchsian space (see [PP06]). Note that the pair $(\sigma, q)$ provides a parameter space of real dimension $16 g-16$, which agrees with the real dimension of complex hyperbolic quasi-Fuchsian space. Equation (1.1) is one of several equations corresponding to immersing a closed surface (or the universal cover) into other geometries. It is of great interest in higher Teichmüller theory to understand the space of surface group representations into higher rank Lie groups, and to integrate techniques of non-linear analysis with the representation theory.

It is also worth mentioning that the Lagrangian property is preserved under the mean curvature flow in Kähler manifolds [Smo96], while minimal surfaces or surfaces of constant mean curvature are often natural candidates for limiting submanifolds (if exist) of various mean curvature flows. The understanding of existence and uniqueness 
of minimal Lagrangian immersions can provide important geometric insight for the analysis of Lagrangian mean curvature flows in Kähler manifolds (see for instance [Wan08]). Minimal Lagrangian submanifolds also play vital roles in the geometry of calibrated submanifolds in Calabi-Yau manifolds [HL82], the SYZ conjecture in mirror symmetry [SYZ96, LYZ05], and symplectic topology [Joy05], to name a few.

Plan of the paper. This paper is organized as follows: In Section 2, after recalling the preliminaries of minimal Lagrangian submanifolds in $\mathbb{C H}^{2}$, we set up the structure equations and reduce the minimal Lagrangian immersion problem to the solutions to equation (1.1) (Proposition 2.1), and we relate the second fundamental form of the minimal Lagrangian immersion to the prescribed conformal structure and holomorphic cubic differential (Proposition 2.2). We prove part (iii) of Theorem 1.1 in Section 3.1, and prove the existence of a solution curve $\gamma$ in Section 3.2. In Section 3.3, we derive a uniform estimate for the solutions on the solution curve away from zero, and hence show the right endpoint $T_{0}$ is in fact included on the solution curve $\gamma$. We then complete the proof of Theorem 1.1 in Section 4, where we focus on the non-uniqueness of minimal Lagrangian immersions with prescribed data. In Section 5, since the solutions near the trivial solution $\gamma(0)$ are unique, we are able to define a functional on a subspace of the space of minimal Lagrangian immersions in $\mathbb{C H}^{2}$. As an application to Teichmüller theory, we show this functional is a potential function of the Weil-Petersson norm of holomorphic cubic differentials.

\section{Minimal Lagrangian submanifolds in $\mathbb{C H}^{2}$}

2.1. Complex hyperbolic space. Before we move to our main interest in $\mathbb{C H}^{2}$, let us briefly mention a few general facts on minimal Lagrangian submanifolds in a Kähler manifold. Let $\left(M^{2 n}, \omega\right)$ be a Kähler manifold where $\omega$ is its Kähler form. Let $N^{n}$ be a submanifold of dimension $n$ in $M^{2 n}$. The inclusion map $i: N^{n} \rightarrow M^{2 n}$ is called Lagrangian if

$$
i^{*} \omega \equiv 0 .
$$

In other words, a Lagrangian submanifold is characterized by the vanishing of $\left.\omega\right|_{N}$. In terms of the Riemannian structure on $M^{2 n}$, the submanifold $N^{n}$ is Lagrangian if the tangent space $T M$ restricted on $N^{n}$ is an orthogonal direct sum as follows:

$$
T M_{N}=T N \oplus J \cdot T N
$$

where $J$ is the complex structure on $M^{2 n}$ such that the Riemannian metric on $M^{2 n}$ is given by $\omega(X, J Y)$ for tangent vectors $X$ and $Y$.

A Lagrangian submanifold $N^{n}$ is minimal if its mean curvature vector is identically zero. Similar to the minimal surface case, these minimal submanifolds are critical points of the volume with respect to induced measure from $M^{2 n}$ [Sim68].

We now consider the space $\mathbb{C H}^{2}$, and use the projective model. Consider the inner product $\langle\cdot, \cdot\rangle$ on $\mathbb{C}^{2,1}$ as follows:

$$
\langle v, w\rangle=v_{1} \bar{w}_{1}+v_{2} \bar{w}_{2}-v_{3} \bar{w}_{3} .
$$


We denote the cone $W_{-}=\left\{v \in \mathbb{C}^{2,1}:\langle v, v\rangle<0\right\}$, and let $P$ be the natural projection $\mathbb{C}^{2,1} \backslash\{0\} \rightarrow \mathbb{C P}^{2}$. Then we define $\mathbb{C H}^{2}$ as a complex manifold to be the image of

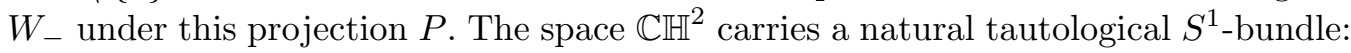
the pseudo-sphere

$$
S_{-}=\left\{u \in W_{-}:\langle u, u\rangle=-1\right\}
$$

and $-\langle\cdot, \cdot\rangle$ induces a metric connection on the $S^{1}$-bundle: $\pi: S_{-} \rightarrow \mathbb{C H}^{2}$.

Note that $\mathbb{C H}^{2}$ does not admit any real totally geodesic hypersurface, but there are two kinds of totally geodesic co-dimension two subspaces (see [Gol99]), namely, the complex line of constant curvature -1 , and the real Lagrangian plane $\mathbb{R} \mathbb{H}^{2}$ of constant curvature $-\frac{1}{4}$. All minimal Lagrangian immersions we consider here are from the universal cover of a closed surface into $\mathbb{C H}^{2}$. Equation (1.1) has a trivial solution, when $t=0, u=0$. This trivial solution corresponds to the totally geodesic Lagrangian embedding of $\mathbb{R} \mathbb{H}^{2}$ in $\mathbb{C H}^{2}$, and the corresponding representations of the surface group are Fuchsian. Any solution $(u(t), t)$ obtained near $(0,0)$ gives rise to complex hyperbolic quasi-Fuchsian representations, as seen in [LM13]. The geometry of representations for solutions $(u(t), t)$ outside a neighborhood of $(0,0)$ is unclear.

2.2. The structure equation and the reconstruction. In this subsection, we briefly recall the derivation and setup of the structure equations for minimal Lagrangian surfaces in $\mathbb{C H}^{2}$.

Let $D=\tilde{\Sigma}=\{z \in \mathbb{C}:|z|<1\}$ be the unit disk, the universal cover of the surface $\Sigma$, then for any Lagrangian immersion $\varphi: D \rightarrow \mathbb{C H}^{2}$, it admits a horizontal Legendrian lift $f: \tilde{\Sigma} \rightarrow S_{-}$. This lift gives rise to a frame, for any $z \in D$ :

$$
F=\left(f_{z} /\left|f_{z}\right| f_{\bar{z}} /\left|f_{\bar{z}}\right| f\right) .
$$

This frame $F(z)$ lies in $U(2,1)$. Furthermore, it is shown in [LM13] that $F$ lies in $S U(2,1)$ if and only if $\varphi$ is a minimal Lagrangian immersion.

Let $\varphi$ be a conformal Lagrangian immersion of the unit disk $D$ to $\mathbb{C H}^{2}$. There is a local Legendrian lift $f: D \rightarrow S_{-} \subset \mathbb{C}^{2,1}$ so that

$$
\langle f, f\rangle=-1, \quad\left\langle f, f_{z}\right\rangle=\left\langle f, f_{\bar{z}}\right\rangle=0, \quad\left\langle f_{z}, f_{\bar{z}}\right\rangle=0,
$$

and we write the first fundamental form $\left\langle f_{z}, f_{z}\right\rangle=\left\langle f_{\bar{z}}, f_{\bar{z}}\right\rangle$ as $s^{2}$, then $2 s^{2}|d z|^{2}$ is the local expression of the metric on $D$. For a conformal map, the minimality of $\varphi$ is just the condition for it to be a harmonic map

$$
\left\langle f_{z \bar{z}}, f_{z}\right\rangle=\left\langle f_{z \bar{z}}, f_{\bar{z}}\right\rangle=0 .
$$

Define

$$
q=\left\langle f_{z z z}, f\right\rangle=-\left\langle f_{z z}, f_{\bar{z}}\right\rangle,
$$

and we may compute by taking $z$ and $\bar{z}$ derivatives of the above equations to find:

$$
\left\langle f_{z z}, f_{z}\right\rangle=2 s s_{z}, \quad\left\langle f_{\bar{z} \bar{z}}, f_{\bar{z}}\right\rangle=2 s s_{\bar{z}}, \quad\left\langle f_{\bar{z} \bar{z}}, f_{z}\right\rangle=\bar{q} .
$$

The frame $F=\left(f_{z} /\left|f_{z}\right| f_{\bar{z}} /\left|f_{\bar{z}}\right| f\right)$ lies in $S U(2,1)$, and we define the MaurerCartan form

$$
\alpha=F^{-1} d F=A d z+B d \bar{z}
$$


where

and

$$
A=F^{-1} F_{z}=\left(\begin{array}{ccc}
(\log s)_{z} & 0 & s \\
-q s^{-2} & -(\log s)_{z} & 0 \\
0 & s & 0
\end{array}\right),
$$

$$
B=F^{-1} F_{\bar{z}}=\left(\begin{array}{ccc}
-(\log s)_{\bar{z}} & \bar{q} s^{-2} & 0 \\
0 & (\log s)_{\bar{z}} & s \\
s & 0 & 0
\end{array}\right) .
$$

The Maurer-Cartan equations $d \alpha+\alpha \wedge \alpha=0$ are equivalent to the following:

$$
\left\{\begin{array}{l}
q_{\bar{z}}=0 \\
\frac{\partial^{2}}{\partial z \partial \bar{z}} \log \left(s^{2}\right)=|q|^{2} s^{-4}+s^{2} .
\end{array}\right.
$$

Now using $\Delta$ as the Laplacian of the hyperbolic metric $g_{\sigma}$ on $(\Sigma, \sigma(z))$, and

$$
\Delta=\frac{4}{g_{\sigma}} \frac{\partial^{2}}{\partial z \partial \bar{z}}, \quad-1=-\frac{2}{g_{\sigma}} \frac{\partial^{2}}{\partial z \partial \bar{z}} \log \left(g_{\sigma}\right) .
$$

Since $e^{u} g_{\sigma}=2 s^{2}$, the second equation of (2.5) becomes the local version of

$$
\Delta u+2-2 e^{u}-16\|q\|^{2} e^{-2 u}=0 .
$$

Since the Maurer-Cartan equations are the integrability conditions for the frame $F$, we have the following local characterization of minimal Lagrangian immersions in $\mathbb{C H}^{2}$ :

Proposition 2.1. A conformal minimal Lagrangian immersion $\varphi: D \rightarrow \mathbb{C H}^{2}$ induces a holomorphic cubic differential $q$ and metric $2 s^{2}|d z|^{2}$ which satisfy (2.5). Conversely, if $\sigma$ is a conformal structure on $\Sigma$ and $q$ is a holomorphic cubic differential on $(\Sigma, \sigma)$. Then any solution $u: \Sigma \rightarrow \mathbb{R}$ to (2.6) determines a Legendrian frame $F: D=\tilde{\Sigma} \rightarrow$ $S U(2,1)$ for a minimal Lagrangian immersion $\varphi: D \rightarrow \mathbb{C H}^{2}$ which is equivariant with respect to some surface group representation from $\pi_{1}(\Sigma)$ into $S U(2,1)$. The immersed minimal Lagrangian surface is unique up to holomorphic isometries of $\mathbb{C H}^{2}$.

This Proposition provides the reconstruction scheme from solving (2.6): Given a conformal structure $\sigma$ on the closed surface $\Sigma$, and a holomorphic cubic differential $q$ on $(\Sigma, \sigma)$, each solution $u(z)$ of equation (2.6) corresponds to an induced metric $2 s^{2}|d z|^{2}$ on $D$. From the formula in (2.4), the 1 -form $\alpha$ is therefore determined. As in [LM13], one integrates the Maurer-Cartan equations $F^{-1} d F=\alpha$ to obtain a frame $F=\left(f_{z} /\left|f_{z}\right| f_{\bar{z}} /\left|f_{\bar{z}}\right| f\right)$. The Lagrangian and minimal properties are encoded in verifying $F \in S U(2,1)$. Therefore, we reduce the problem of obtaining surface group equivariant minimal Lagrangian immersion of the disk $D$ into $\mathbb{C H}^{2}$ to the solvability of equation $(2.6)$ for data $(\sigma, q)$.

2.3. The second fundamental form. For any immersion problem, the second fundamental form is a natural key object of the study, and they often give rise to the concepts of principal curvatures. In the case of a minimal surface in a hyperbolic three-manifold, the second fundamental form is described as the real part of a holomorphic quadratic differential. In this subsection, we show that an analogous fact is true for minimal Lagrangian surfaces in $\mathbb{C H}^{2}$, namely, we describe all the components 
of the second fundamental form in terms of real and imaginary parts of a holomorphic cubic differential $q$.

To compute the second fundamental form, we consider for a conformal coordinate $z=x+i y$ the orthonormal basis of the tangent space of the immersed surface given by $E_{1}=f_{x} /\left|f_{x}\right|, E_{2}=f_{y} /\left|f_{y}\right|$. Since the surface is Lagrangian, by (2.1), $\left(E_{1}, E_{2}, i E_{1}, i E_{2}\right)$ form an orthonormal basis in the tangent space of $\mathbb{C H}^{2}$. For tangent vector fields $X, Y \in \Gamma(T \varphi(D))$, the second fundamental form of the immersion into $\mathbb{C H}^{2}$ is given by

$$
\mathbb{I}(X, Y)=\sum_{j=1}^{2} g\left(\nabla_{X} Y, i E_{j}\right) i E_{j},
$$

where $g(v, w)$ is the Riemannian metric on $\mathbb{C H}^{2}$ inherited from the inner product $(2.2)$ on $\mathbb{C}^{2,1}$, and $\nabla$ is the Levi-Civita connection on $\mathbb{C H}^{2}$, which is the projection of the flat connection on $\mathbb{C}^{2,1}$.

We find that there are three independent entries for the second fundamental form II, which allows us to arrange them in a $2 \times 2$ symmetric matrix:

Proposition 2.2. All the components of II are determined by the metric $2 s^{2}|d z|^{2}$ and the cubic differential $q$. In particular, we have

$$
\begin{aligned}
& \mathbb{I}\left(E_{1}, E_{1}\right)=2^{-\frac{1}{2}} s^{-3}\left(-\operatorname{Im} q \cdot i E_{1}-\operatorname{Re} q \cdot i E_{2}\right), \\
& \mathbb{I}\left(E_{1}, E_{2}\right)=2^{-\frac{1}{2}} s^{-3}\left(-\operatorname{Re} q \cdot i E_{1}+\operatorname{Im} q \cdot i E_{2}\right), \\
& \mathbb{I}\left(E_{2}, E_{2}\right)=2^{-\frac{1}{2}} s^{-3}\left(\operatorname{Im} q \cdot i E_{1}+\operatorname{Re} q \cdot i E_{2}\right) .
\end{aligned}
$$

Proof. We compute only $\mathbb{I}\left(E_{1}, E_{1}\right)$, as the rest are similar. Since the Levi-Civita connection on $\mathbb{C H}^{2}$ is the projection of the flat connection on $\mathbb{C}^{2,1}$, we can compute $\nabla_{X} Y$ as $(X Y) f$. Therefore,

$$
\begin{aligned}
\mathbb{I}\left(E_{1}, E_{1}\right) & =\frac{1}{2 s^{2}} \mathbb{I}\left(f_{x}, f_{x}\right) \\
& =\sum_{j=1}^{2} \frac{1}{2 s^{2}} g\left(f_{x x}, i E_{j}\right) \cdot i E_{j} \\
& =\frac{1}{2 \sqrt{2} \cdot s^{3}}\left[g\left(f_{x x}, i f_{x}\right) \cdot i E_{1}+g\left(f_{x x}, i f_{y}\right) \cdot i E_{2}\right]
\end{aligned}
$$

Now compute

$$
\begin{aligned}
g\left(f_{x x}, i f_{x}\right) & =\operatorname{Re}\left\langle f_{z z}+2 f_{z \bar{z}}+f_{\bar{z} \bar{z}}, i f_{z}+i f_{\bar{z}}\right\rangle \\
& =\operatorname{Re}\left[-i\left(2 s s_{z}-q+\bar{q}+2 s s_{\bar{z}}\right)\right] \\
& =-2 \operatorname{Im} q .
\end{aligned}
$$

We may similarly compute $g\left(f_{x x}, i f_{y}\right)=-2 \operatorname{Re} q$. So altogether,

$$
\mathbb{I}\left(E_{1}, E_{1}\right)=\frac{1}{2 \sqrt{2} \cdot s^{3}}\left[-2 \operatorname{Im} q \cdot i E_{1}-2 \operatorname{Re} q \cdot i E_{2}\right] .
$$




\section{General existence results}

We now consider a family of equations (2.6) determined the ray $t q(t \geq 0)$ in the space of holomorphic cubic differentials $C(\sigma)$ :

$$
\Delta u(z, t)+2-2 e^{u(z, t)}-16 t^{2}\|q\|^{2} e^{-2 u(z, t)}=0 .
$$

Recall from (1.2) that $\|q\|^{2}=\frac{q \bar{q}}{g_{\sigma}^{3}}$.

It is an immediate consequence of the maximum principle that we have:

Proposition 3.1. Any solution $u$ to (3.1) satisfies $u \leq 0$.

Since $C(\sigma)$ is a finite dimensional vector space, this approach allows us to fix $q$ and focus on finding interval of the parameter $t$ for which solutions to (3.1) exists. This setup is quite standard in non-linear analysis, where we have access to several important techniques such as the continuity method and the variational method. Equations (1.1) and (3.1) are very similar to the equations in the problem of minimal immersions of closed surfaces into hyperbolic three-manifolds first introduced by Uhlenbeck [Uhl83] and further studied in [HL12].

3.1. Nonexistence. In this subsection, we deal with (possibly) large values for parameter $t$.

Theorem 3.2. There exists a constant $T=T(q, \sigma)$ such that equation (3.1) does not admit any solution for any $t \geq T$.

Note that, the solvability of equation (3.1) is a necessary condition for the existence of a minimal Lagrangian immersion of $\Sigma$ into $\mathbb{C H}^{2}$. This theorem is equivalent to Proposition 5.8 in [LM13]. We include a proof here for the sake of completeness since it is very short.

Proof. We integrate equation (3.1) with respect to the hyperbolic metric $g_{\sigma}$ :

$$
A_{\sigma}=8 t^{2} \int_{\Sigma}\|q\|^{2} e^{-2 u} d A_{\sigma}+\int_{\Sigma} e^{u} d A_{\sigma}>8 t^{2} \int_{\Sigma}\|q\|^{2} e^{-2 u} d A_{\sigma} .
$$

Meanwhile, we apply the Hölder's inequality, and Proposition 3.1:

$$
\begin{aligned}
\int_{\Sigma}\|q\|^{2 / 3} d A_{\sigma} & =\int_{\Sigma}\|q\|^{2 / 3} e^{-2 u / 3} e^{2 u / 3} d A_{\sigma} \\
& \leq\left\{\int_{\Sigma}\|q\|^{2} e^{-2 u} d A_{\sigma}\right\}^{1 / 3}\left\{\int_{\Sigma} e^{u} d A_{\sigma}\right\}^{2 / 3} \\
& \leq A_{\sigma}^{2 / 3}\left\{\int_{\Sigma}\|q\|^{2} e^{-2 u} d A_{\sigma}\right\}^{1 / 3} .
\end{aligned}
$$

Applying above inequality to (3.2), and noting that the hyperbolic area of $\Sigma$ is $A_{\sigma}=2 \pi(2 g-2)$, we find:

$$
t<\left\{\frac{2 \pi(g-1)}{\int_{\Sigma}\|q\|^{2 / 3} d A_{\sigma}}\right\}^{3 / 2} .
$$

Now we can simply choose $T=\left\{\frac{2 \pi(g-1)}{\int_{\Sigma}\|q\|^{2 / 3} d A_{\sigma}}\right\}^{3 / 2}$. 
3.2. Existence. In this subsection, we take advantage of this variational setup to apply the implicit function theorem to prove the solvability of equation (3.1) and therefore the general existence of minimal Lagrangian immersion of $\tilde{\Sigma}$ into $\mathbb{C H}^{2}$.

We consider the non-linear map $F: W^{2,2}(\Sigma) \times[0, \infty) \rightarrow L^{2}(\Sigma)$ defined by

$$
F(u, t)=\Delta u+2-2 e^{u}-16 t^{2}\|q\|^{2} e^{-2 u},
$$

where $W^{2, k}(\Sigma)$ stands for the classical Sobolev space. At each $t \geq 0$ fixed, the linearized operator $L(u, t): W^{2,2}(\Sigma) \rightarrow L^{2}(\Sigma)$ associated to $F$ is given by

$$
L(u, t)=-\Delta+2 e^{-2 u}\left(e^{3 u}-16 t^{2}\|q\|^{2}\right) .
$$

It is easy to see that $L(u, 0)>0$, since $-\Delta$ has non-negative eigenvalues. In standard theory, the operator $L(u, t)$ in (3.4) is crucial in order to apply the implicit function theorem. In particular, when the linearized operator $L$ has all positive eigenvalues, the differential of the map $F(u, t)$ in $(3.3)$ is onto.

The existence of solutions for small $t$ is implied by the following:

Theorem 3.3. There exists a constant $T_{0}=T_{0}(\sigma, q)>0$ and a smooth curve

$$
\gamma:\left[0, T_{0}\right] \rightarrow W^{2,2}(\Sigma) \times[0, \infty) \quad t \mapsto(u(t), t),
$$

such that

(a) $\gamma(0)=(0,0)$ and $F(\gamma(t))=0$ for all $t \in\left[0, T_{0}\right]$.

(b) $L(u(t), t)>0$ for all $t \in\left[0, T_{0}\right)$.

(c) $\operatorname{Ker}\left(L\left(u\left(T_{0}\right), T_{0}\right)\right) \neq\{0\}$.

(d) The family of solutions to $F(u(t), t)=0$ is unique near $\gamma(0)=(0,0)$.

Proof. We follow closely the existence of solutions in [Uhl83].

We use the continuity method. Let $E=\{t \in[0, \infty)$ : there is a unique smooth solution $\gamma(\tau)$ with initial condition $\gamma(0)=(0,0)$ and which satisfies $F(\gamma(\tau))=0$ and $L(u(\tau), \tau)>0$ for all $\tau \in[0, t]\}$. Clearly $E$ includes 0 . $E$ is open by the implicit function theorem and since $L(u(t), t)>0$. To see the closedness, apply Theorem 3.7 below and standard elliptic theory to find that $E$ contains any $t \in \bar{E}$ so that $\operatorname{Ker}(L(u(t), t))=\{0\}$. Therefore the statements (a) (b) and (d) follow. Statement (c) then follows by the estimate in Theorem 3.7.

Remark 3.4. (i) Note that by standard regularity theory, the solution $u(t)$ obtained above belongs to the class $C^{\infty}(\Sigma)$.

(ii) One can show that for $t^{2}\|q\|^{2} \leq \frac{1}{54}$, a lower bound on $u$ along $\gamma$ from [LM13] implies that $L$ is a positive operator, and thus we find an alternate proof of the existence result in [LM13].

3.3. Estimates for closedness. We start with a lemma that we will use later:

Lemma 3.5. For $a \geq 1$ and $b \geq 0$, we have

$$
a b \leq H(a)+H^{*}(b),
$$

where $H(a)=\frac{1}{4} a(\log a)^{2}$ and $H^{*}(b)=\frac{1}{2} e^{-1+\sqrt{1+4 b}}(-1+\sqrt{1+4 b})$. 
Proof. For $a \geq 1, H(a)$ is a convex function. Then we may compute

$$
H^{*}(b)=\sup _{a \geq 1}[a b-H(a)]
$$

as the Legendre transform of $H$. Indeed, we have

$$
H^{\prime}(a)=\frac{1}{4}(\log a)^{2}+\frac{1}{2} \log a,
$$

and consequently, for $b=H^{\prime}(a)$,

$$
H^{*}(b)=H^{*}\left(\frac{1}{4}(\log a)^{2}+\frac{1}{2} \log a\right)=a H^{\prime}(a)-H(a)=\frac{1}{2} a \log a .
$$

It is then easy to solve for the formula of $H^{*}(b)$.

We are left to show the following key estimate to complete the proof of Theorem 3.3, namely, the solutions on $t \in\left[0, T_{0}\right)$ given by the implicit function theorem can be extended to $t=T_{0}$.

Remark 3.6. The following type of estimate is known to Uhlenbeck [Uhl83, p. 164], but the proof is not included in [Uhl83].

Theorem 3.7. Let $p \in(1, \infty)$. Then there is a constant $C=C(\sigma, q, p)$ such that for every solution $u$ of along the path $\gamma$ in Theorem 3.3 from $t=0$ satisfying $F(u, t)=0$ and $L(u, t) \geq 0$, we have

$$
\|u\|_{W^{2, p}} \leq C .
$$

Proof. By the implicit function theorem around $t=0$, we may assume there is a fixed $\epsilon>0$ so that $t \geq \epsilon$. Now we assume $u$ is a solution on the solution curve $\gamma$ with $L(u, t) \geq 0$, and we integrate both sides of (3.1) with respect to the hyperbolic metric on $\Sigma$ to find:

$$
A_{\sigma}=\int_{\Sigma} e^{u}+8 t^{2} \int_{\Sigma}\|q\|^{2} e^{-2 u}
$$

where we recall that $A_{\sigma}=2 \pi(2 g-2)$ is the hyperbolic area of $\Sigma$.

Since $t \geq \epsilon$, we have for a positive constant $C_{1}=C_{1}(\epsilon)$,

$$
\int_{\Sigma} e^{-2 u}\|q\|^{2} \leq C_{1}
$$

Since $q$ is a prescribed holomorphic cubic differential on a closed surface $(\Sigma, \sigma)$, it is well-known that $q$ has isolated zeros.

To derive an integral bound on $u$, let $n$ be the largest order of all the zeros of $q$, and let $\ell<\frac{1}{n+1}, \alpha=\frac{1}{\ell}$ and $\beta$ be the conjugate exponent of $\alpha$ (namely $\frac{1}{\alpha}+\frac{1}{\beta}=1$ ). Now $\left\|q^{-2 \ell}\right\|_{\beta}$ is finite, which allows us to apply Hölder's Inequality:

$$
\int_{\Sigma}\left(e^{-2 u}\right)^{\ell} \leq\left\|\left(e^{-2 u}\|q\|^{2}\right)^{\ell}\right\|_{\alpha} \cdot\left\|q^{-2 \ell}\right\|_{\beta}<C_{2},
$$

for some $C_{2}=C_{2}(\sigma, q, \epsilon)>0$.

Since $u \leq 0$ (Proposition 3.1), each $|u|^{p}$ is dominated by $e^{-2 \ell u}$, and we have uniform $L^{p}$ bounds, for any $p>1$,

$$
\|u\|_{p} \leq C_{3}=C_{3}(\sigma, q, \epsilon, p)
$$


Furthermore, since $\langle L(u, t) u, u\rangle \geq 0$, we have

$$
\begin{aligned}
\langle L(u, t) u, u\rangle & =\int_{\Sigma}\left(-\Delta u+2 e^{-2 u}\left(e^{3 u}-16 t^{2}\|q\|^{2}\right) u\right) u \\
& =\int_{\Sigma}|\nabla u|^{2}+2 e^{u} u^{2}-32 e^{-2 u} t^{2}\|q\|^{2} u^{2} \geq 0 .
\end{aligned}
$$

We then multiply equation (3.1) by $u$ and integrate by parts to find:

$$
\int_{\Sigma}|\nabla u|^{2}=\int_{\Sigma} 2 u-2 u e^{u}-16 t^{2}\|q\|^{2} u e^{-2 u} .
$$

Applying (3.9) to the inequality (3.8), we have

$$
\int_{\Sigma} 2 u+2 e^{u}\left(u^{2}-u\right)-16 t^{2}\|q\|^{2} e^{-2 u}\left(2 u^{2}+u\right) \geq 0 .
$$

Now the combination of $u \leq 0$ and the $L^{p}$-bound (3.7) gives the following:

$$
\int_{\Sigma} 16 t^{2}\|q\|^{2} e^{-2 u} u^{2} \leq C_{4}
$$

for some uniform constant $C_{4}=C_{4}(\sigma, q, \epsilon, p)>0$. We note the extra $u^{2}$ in this integral, together with the Green's function representation of solutions to (3.1), will be enough to prove uniform $L^{\infty}$-estimates on $u$.

To proceed, we define the following simplified notion for two functions $f$ and $g$ : $f=g+O(1)$ if $|f-g|$ is uniformly bounded from above by some positive constant.

Let $G(x, y)$ be the Green's function for the hyperbolic Laplacian. We have

$$
G(x, y)=\frac{1}{2 \pi} \log (d(x, y))+O(1)
$$

for $d$ the hyperbolic distance.

Let $\bar{u}$ be the average of $u$, which is bounded by the $L^{p}$-bound (3.7). We apply the Green's formula to find:

$$
\begin{aligned}
u(x) & =\bar{u}+\int_{\Sigma} G(x, y) \Delta u(y) d \operatorname{Vol}(y) \\
& =\int_{\Sigma} G(x, y)\left[-2+2 e^{u(y)}+16 t^{2}\|q(y)\|^{2} e^{-2 u(y)}\right] d \operatorname{Vol}(y)+O(1) \\
& =16 t^{2} \int_{\Sigma} G(x, y)\|q(y)\|^{2} e^{-2 u(y)} d \operatorname{Vol}(y)+O(1) .
\end{aligned}
$$

Now choose a complex normal coordinate disk $\mathcal{D}$ centered at $x$ (so that $x=0$ ), and use the asymptotics of the Green's function, together with (3.6), to find

$$
u(0)=\frac{8 t^{2}}{\pi} \int_{\mathcal{D}} \log |y| e^{-2 u}\|q\|^{2} d \operatorname{Vol}(y)+O(1) .
$$


We want to compare this integral to $\int t^{2} e^{-2 u} u^{2}\|q\|^{2}$, for which we have a bound by (3.11). We now choose $a=e^{-2 u}$ and $b=|\log | y||$. It is easy to verify that $a \geq 1$ and $b=|\log | y|| \geq 0$. Hence $H(a)=u^{2} e^{-2 u}$ and $H^{*}(b)=\frac{1}{2} e^{-1+\sqrt{1+4 b}}(-1+\sqrt{1+4 b})$. We can now apply Lemma 3.5 to find:

$$
\begin{aligned}
|u(0)| & \leq \frac{8 t^{2}}{\pi} \int_{\mathcal{D}}|\log | y|| \cdot e^{-2 u} \cdot\|q\|^{2} d V o l(y)+O(1) \\
& \leq \frac{8 t^{2}}{\pi} \int_{\Sigma} e^{-2 u} u^{2}\|q\|^{2}+\frac{8 t^{2}}{\pi} \int_{\mathcal{D}} H^{*}(b)\|q\|^{2} d V o l(y)+O(1) .
\end{aligned}
$$

Both these terms are bounded, the first by (3.11), and the second by a direct computation. Since $0=x \in \Sigma$ was arbitrary, we have a uniform bound

$$
\|u\|_{L^{\infty}} \leq C_{5}
$$

This bound can then be plugged into the equation $F(u, t)=0$ to find uniform $L^{\infty}$ bounds on $\Delta u$. Thus standard $L^{p}$ theory applies, and we have uniform $W^{2, p}$ bounds on $u$. Higher regularity is standard.

\section{Non-uniqueness}

In previous sections, we have proved parts (ii) and (iii) of Theorem 1.1. By the implicit function theorem, the family of solutions to the structure equation is unique for the family including $\gamma(0)$. In this section, we address the issue of non-uniqueness for this problem, i.e., we construct a mountain pass type solution for each parameter value $t$ on $\left(0, T_{0}\right)$. This will complete the proof of part (i).

4.1. New formulation. We start with a new formulation of the problem in order to prove a compactness result. This is necessary because the original Euler-Lagrange functional associated to the structure equation (3.1) does not satisfy a compactness property that is required to apply the mountain pass theorem. Our approach is to follow the strategy used in [HL12] for the minimal immersion problem in hyperbolic three-manifolds: we define a new functional and a new norm for the structure equation (3.1), and show the critical points of the new functional coincide with the solutions of (3.1), and in next subsection we prove a compactness theorem for the new functional and norm, and finally apply the mountain pass theorem in [AR73] to produce a second solution for each $t$ on $\left(0, T_{0}\right)$.

To proceed, we need to capture the non-linearities arising from the structure equation (3.1), which now we recall:

$$
\Delta u+2-2 e^{u}-V e^{-2 u}=0,
$$

where we set $V=V(t, z)=16 t^{2}\|q\|^{2}$.

Let $H^{1}(\Sigma)$ be the usual Sobolev space

$$
H^{1}(\Sigma):=\left\{u \in L^{2}(\Sigma): \nabla u \in L^{2}(\Sigma)\right\},
$$

equiped with the norm

$$
\langle f, g\rangle_{H^{1}}:=\int_{\Sigma}\{\nabla f \nabla g+f g\} .
$$


Then for $u \in H^{1}(\Sigma)$, the Euler-Lagrange functional for (3.1) is

$$
I(u):=\frac{1}{2} \int_{\Sigma}|\nabla u|^{2}+\int_{\Sigma}\left(2 e^{u}-2 u-V \frac{e^{-2 u}}{2}\right) .
$$

Note that this functional does not satisfy the Palais-Smale compactness condition.

We now explicitly construct smooth functions as follows:

$$
f_{1}(s):=\left\{\begin{array}{ll}
2-2 e^{s} & \text { if } s \leq 0 \\
-\theta s^{\theta-1} & \text { if } s>1
\end{array},\right.
$$

where $\theta>2$ is a constant and we require $f_{1}(s)<0$ for $s>0$. Let

$$
f_{2}(s):= \begin{cases}s-e^{-2 s} & \text { if } s \leq 0 \\ 0 & \text { if } s>1 .\end{cases}
$$

In addition, it is easy to see that we can also require $f_{2}(s)<0$ for all $s \in(0,1)$.

With these functions, we can transfer equation (3.1) to a new equation which is better suited for variational methods:

Lemma 4.1. The structure equation (3.1) is equivalent to the new equation

$$
-\Delta u+V u-\left(f_{1}(u)+V f_{2}(u)\right)=0 .
$$

Proof. First, let $u$ be a solution to equation (3.1). Then from Proposition 3.1, we have $u \leq 0$. In this case, from the explicit formulas in (4.2) and (4.3), we find that $f_{1}(u)=2-2 e^{u}$ and $f_{2}(u)=u-e^{-2 u}$.

We now verify that

$$
\begin{aligned}
-\Delta u+V u-f_{1}(u)-V f_{2}(u) & =-\Delta u+V u-2+2 e^{u}-V(z) u+V e^{-2 u} \\
& =-\Delta u-2+2 e^{u}+V e^{-2 u} \\
& =0 .
\end{aligned}
$$

Conversely, let $u$ be a solution to equation (4.4). We now apply the maximum principle to (4.4). At the maximum point $p_{0}$ of $u$, we have $\Delta u\left(p_{0}\right) \leq 0$. Note that these functions $f_{1}$ and $f_{2}$ enjoy the following properties:

$$
f_{1}(s)<0 \quad \forall s>0, \quad f_{2}(s) \leq \min \{0, s\} \quad \forall s \in \mathbb{R} .
$$

Therefore at $p_{0}$, we have either $u\left(p_{0}\right) \leq 0$ or

$$
V u\left(p_{0}\right) \leq f_{1}\left(u\left(p_{0}\right)\right)+V(z) f_{2}\left(u\left(p_{0}\right)\right) \leq f_{1}\left(u\left(p_{0}\right)\right)+V u\left(p_{0}\right) .
$$

This implies that $f_{1}\left(u\left(p_{0}\right)\right) \geq 0$, and therefore $u\left(p_{0}\right) \leq 0$ by the definition (4.2) of $f_{1}$. Since $p_{0}$ is the maximum of $u$, we have just showed $u \leq 0$. It is then easy to see that the sets of solutions of (3.1) and (4.4) coincide.

To define the functional for this new equation (4.4), we need to introduce an appropriate norm on $H^{1}(\Sigma)$ as follows:

$$
\langle f, g\rangle_{V}:=\int_{\Sigma}\{\nabla f \nabla g+V(z) f g\} .
$$

Lemma 4.2. The norms $\|\cdot\|_{H^{1}},\|\cdot\|_{V}$ on the Sobolev space $H^{1}(\Sigma)$ are equivalent. 
Proof. One direction is obvious, namely, since $V \in L^{\infty}(\Sigma)$, we have $\|\cdot\|_{V} \leq C\|\cdot\|_{H^{1}}$. To work in the opposite direction, we set $\bar{u}:=\frac{1}{A_{\sigma}} \int_{\Sigma} u$ as the average of $u$ on $\Sigma$, where $A_{\sigma}=4 \pi(g-1)$ is the hyperbolic area of the surface.

We start with

$$
\|u\|_{L^{2}} \leq\|u-\bar{u}\|_{L^{2}}+\|\bar{u}\|_{L^{2}},
$$

and the first term on the right-hand side of (4.6) is taken care of by the Poincaré inequality, namely, we have

$$
\|u-\bar{u}\|_{L^{2}} \leq C\|\nabla u\|_{L^{2}} .
$$

For the second term, we use

$$
|\bar{u}|^{2} \leq 2 u^{2}+2|u-\bar{u}|^{2}
$$

and apply Poincaré inequality again to find:

$$
\begin{aligned}
|\bar{u}|^{2} \int_{\Sigma} V(z) & \leq 2 \int_{\Sigma}\left\{V(z)|\bar{u}-u|^{2}+V(z) u^{2}\right\} \\
& \leq C \int_{\Sigma}\left\{|\bar{u}-u|^{2}+V(z) u^{2}\right\} \\
& \leq C \int_{\Sigma}\left\{|\nabla u|^{2}+V(z) u^{2}\right\} \\
& =C\|u\|_{V}^{2} .
\end{aligned}
$$

Since $\int_{\Sigma} V>0$, we also bound the second term in the right-hand side of (4.6), and complete the proof.

We integrate from the formulas (4.2) and (4.3) to define new functions:

$$
F_{1}(s):= \begin{cases}2 s-2 e^{s}+2 & \text { if } s \leq 0 \\ -s^{\theta} & \text { if } s>1\end{cases}
$$

and

$$
F_{2}(s):= \begin{cases}\frac{1}{2}\left(s^{2}+e^{-2 s}\right) & \text { if } s \leq 0 \\ 0 & \text { if } s>1\end{cases}
$$

Note that the additive constant 2 in the formula of $F_{1}(s)$ when $s \leq 0$ is designed such that $f_{1}(s)=F_{1}^{\prime}(s)<0$ when $s>0$. Using these functions, we can now define the functional corresponding to the formulation in equation (4.4), on the Hilbert space $H^{1}(\Sigma)$, as the following:

$$
\mathcal{F}(u):=\frac{1}{2} \int_{\Sigma}\left\{|\nabla u|^{2}+V(z) u^{2}\right\}-\int_{\Sigma}\left\{F_{1}(u)+V(z) F_{2}(u)\right\}, \quad u \in H^{1}(\Sigma),
$$

This functional is well-defined by the Moser-Trudinger inequality, and it is clear that it is continuously differentiable.

Remark 4.3. From this definition (4.10), the critical points of $\mathcal{F}$ are weak solutions of (4.4), and hence solutions to the structure equation (3.1) by Lemma 4.1. Also by Lemma 4.1 , for each $t \in\left(0, T_{0}\right)$, Theorem 3.3 provides a critical point of $\mathcal{F}$ which is stable. Our next subsection is to show for each stable critical point of $\mathcal{F}$, there is another solution (of mountain pass type) corresponding to the same $t \in\left(0, T_{0}\right)$. 
We end this subsection by the following easy observation:

Proposition 4.4. Let $k$ be a negative constant, then we have

$$
\lim _{k \rightarrow-\infty} \mathcal{F}(k)=-\infty .
$$

4.2. Strong non-uniqueness. One more step remains before we can prove the (strong) non-uniqueness for the minimal Lagrangian immersion with $(\sigma, t q)$, where $\sigma \in \mathcal{T}_{g}(\Sigma)$ and $q \in C(\sigma)$, for each $t \in\left(0, T_{0}\right)$, namely, we have to show a compactness property for the functional $\mathcal{F}$ :

Theorem 4.5. The functional $\mathcal{F}$ in (4.10) satisfies the Palais-Smale compactness condition.

Proof. Let us facilitate with the following notation. Let $O(1)$ as before, and we call a quantity $|f|=o(1)$ if $|f|$ tends to zero when an appropriate limit is taken. Using this notation, the Palais-Smale compactness condition for the functional $\mathcal{F}$ is equivalent to showing that any sequence of functions $\left\{u_{n} \in H^{1}(\Sigma)\right\}$ which satisfies

$$
\left|\mathcal{F}\left(u_{n}\right)\right|=O(1), \quad\left\|\mathcal{F}^{\prime}\left(u_{n}\right)\right\|_{H^{-1}}=o(1),
$$

admits a subsequence which converges strongly in $H^{1}(\Sigma)$.

Suppose $\left\{u_{n} \in H^{1}(\Sigma)\right\}$ is a sequence which satisfies (4.12), and we will prove the theorem in two steps: first we show $\left\{u_{n}\right\}$ is bounded, hence there is a weak limit in $H^{1}(\Sigma)$, then we show this weak limit is actually strong.

Step one: $\left\|u_{n}\right\|_{V}=O(1)$.

To see this, we deduce from the expressions of the functional $\mathcal{F}$ in $(4.10)$, the norm $\|\cdot\|_{V}$ in (4.5), and the assumption that $\mathcal{F}\left(u_{n}\right)=O(1)$, we have

$$
\frac{1}{2}\left\|u_{n}\right\|_{V}^{2} \leq \int_{\Sigma}\left\{F_{1}\left(u_{n}\right)+V F_{2}\left(u_{n}\right)\right\}+O(1) .
$$

It is not hard to verify, from the definitions of functions $f_{j}(s)$ and $F_{j}(s), j=1,2$, by considering all three subintervals for $s \in \mathbb{R}:(-\infty, 0),[0,1]$ and $(1, \infty)$, that, for $\theta>2$,

$$
F_{j}(s) \leq \frac{s}{\theta} f_{j}(s)+O(1), \quad j=1,2 .
$$

On the other hand, in the direction of $\xi$, we have

$$
\mathcal{F}^{\prime}(u)(\xi)=\int_{\Sigma}(\nabla u \nabla \xi+V u \xi)-\int_{\Sigma} \xi\left(f_{1}(u)+V f_{2}(u)\right) .
$$

We deduce from the assumption $\left\|\mathcal{F}^{\prime}\left(u_{n}\right)\right\|_{H^{-1}}=o(1)$ that

$$
\mathcal{F}\left(u_{n}\right)\left(u_{n}\right)=o(1)\left\|u_{n}\right\|_{V} .
$$

Therefore we have

$$
\left\|u_{n}\right\|_{V}^{2}=\int_{\Sigma} u_{n}\left\{f_{1}\left(u_{n}\right)+V f_{2}\left(u_{n}\right)\right\}+o(1)\left\|u_{n}\right\|_{V} .
$$


We now continue from the estimate (4.13) to find

$$
\begin{aligned}
\frac{1}{2}\left\|u_{n}\right\|_{V}^{2} & \leq \int_{\Sigma} \frac{u_{n}}{\theta}\left\{f_{1}\left(u_{n}\right)+V f_{2}\left(u_{n}\right)\right\}+O(1) \\
& =\frac{1}{\theta}\left\|u_{n}\right\|_{V}^{2}+O(1)+o(1)\left\|u_{n}\right\|_{V} .
\end{aligned}
$$

Step one is now completed since $\theta>2$ is a constant. Therefore, we obtain, up to a subsequence, $\left\{u_{n}\right\}$ converges weakly in $H^{1}(\Sigma)$ to some $\hat{u}$.

Step two: $\left\{u_{n}\right\}$ converges strongly to $\hat{u}$, namely, $\left\|u_{n}-\hat{u}\right\|_{H^{1}}=o(1)$.

To complete our proof, by the equivalence of two norms (Lemma 4.2), we only have to show $\left\|u_{n}-\hat{u}\right\|_{V}^{2}=o(1)$. Meanwhile, we have

$$
\left\|u_{n}-\hat{u}\right\|_{V}^{2}=\int_{\Sigma}\left\{\left(f_{1}\left(u_{n}\right)+V f_{2}\left(u_{n}\right)\right)\left(u_{n}-\hat{u}\right)\right\}+o(1) .
$$

From the expression of $f_{1}(s)$ in (4.2) and the fact that $H^{1}$ is compactly included in $L^{p}$ for all $p<\infty$ shows that (perhaps going to a further subsequence) we have $\left\{f_{1}\left(u_{n}\right)\right\}$ converges strongly to $f_{1}(\hat{u})$ in $L^{2}(\Sigma)$. Similarly, from the expression of $f_{2}(s)$ in (4.3), and the Moser-Trudinger inequality, we find that $\left\{f_{2}\left(u_{n}\right)\right\}$ converges strongly to $f_{2}(\hat{u})$ in $L^{2}(\Sigma)$. Now step two is completed from (4.16).

We now prove our main theorem of the section.

Theorem 4.6. For each $t \in\left(0, T_{0}\right)$, where $T_{0}$ is defined in Theorem 3.3, for fixed conformal structure $\sigma \in \mathcal{T}_{g}(\Sigma)$, and holomorphic cubic differential $q d z^{3} \in C(\sigma)$, the structure equation (3.1) admits at least two solutions.

Proof. Since equations (3.1) and (4.4) are equivalent, according to Lemma 4.1, and $\mathcal{F}$ is the associated functional to equation (4.4), we only have to show $\mathcal{F}$ admits at least two critical points for each $t \in\left(0, T_{0}\right)$.

From Theorem 3.3, we have $\mathcal{F}$ admits one critical point $(u(t), t)$ such that the linearized operator is positive. Therefore this (stable) solution $u(t)$ obtained in Theorem 3.3 is a local minimizer for the functional $\mathcal{F}$ in the Hilbert space $H^{1}(\Sigma)$. There then exists a ball $B(u(t), r)$ in $H^{1}(\Sigma)$ such that

$$
\inf _{v \in \partial B(u(t), r)} \mathcal{F}(v) \geq \mathcal{F}(u(t)) .
$$

However, the limit in (4.11) indicates that there must be some function $w \in H^{1}(\Sigma)$ such that

$$
w \notin B(u(t), r), \quad \mathcal{F}(w)<\mathcal{F}(u(t)) .
$$

Since the Palais-Smale condition is satisfied by Theorem 4.5, the additional critical point of $\mathcal{F}$ for any $t \in\left(0, T_{0}\right)$ is obtained by applying the Mountain Pass Theorem of Ambrosetti-Rabinowitz [AR73].

Naturally, one is interested in these solutions when $t$ goes to zero. 
Theorem 4.7. Let $\left\{u_{n}\left(t_{n}\right)\right\}$ be a sequence of solutions to the structure equation (3.1). If $t_{n} \rightarrow 0$ as $n \rightarrow \infty$, then along a subsequence, we have

(i) $u_{n} \rightarrow 0$ uniformly; or

(ii) $\left\|u_{n}\right\|_{\infty} \rightarrow \infty$.

Proof. From equation (3.1), we have

$$
-\Delta u_{n}=2-2 e^{u_{n}}-16 t^{2}\|q\|^{2} e^{-2 u_{n}} .
$$

As $t_{n} \rightarrow 0$, possibly up to a subsequence, we have either $\left\|u_{n}\right\|_{H^{1}}=O(1)$ or $\left\|u_{n}\right\|_{H^{1}} \rightarrow$ $\infty$. Let us assume that $\left\|u_{n}\right\|_{H^{1}}=O(1)$ for the moment. In this case, $u_{n}$ converges weakly to some $\hat{u}$. For any $\xi \in C^{\infty}(\Sigma)$, and using (4.17), we find:

$$
\begin{aligned}
\int_{\Sigma} \nabla \hat{u} \nabla \xi \leftarrow \int_{\Sigma} \nabla u_{n} \nabla \xi & =\int_{\Sigma} 2 \xi\left\{1-e^{u_{n}}-8 t^{2}\|q\|^{2} e^{-2 u_{n}}\right\} \\
& \rightarrow \int_{\Sigma} 2 \xi\left\{1-e^{\hat{u}}\right\}
\end{aligned}
$$

after taking $n \rightarrow \infty$. Since $\xi \in C^{\infty}(\Sigma)$ is arbitrary, we have:

$$
-\Delta \hat{u}=2\left(1-e^{\hat{u}}\right) \text {. }
$$

It is then easy to see via the maximum principle that $\hat{u}=0$. By the uniqueness of the solution near $\gamma(0)=(0,0)$ in Theorem $3.3,\left\{u_{n}\right\}$ coincide with the solutions on $\gamma$ as $t_{n} \rightarrow 0$.

Now we consider the other possibility, namely, $\left\|u_{n}\right\|_{H^{1}} \rightarrow \infty$. We need to show $\left\|u_{n}\right\|_{\infty} \rightarrow \infty$. If otherwise, assuming that $\left\|u_{n}\right\|_{\infty}=O(1)$, we integrate from (4.17) to obtain

$$
\int_{\Sigma}\left|\nabla u_{n}\right|^{2}=\int_{\Sigma} u_{n}\left\{2-2 e^{u_{n}}-V(z) e^{-2 u_{n}}\right\}=O(1) .
$$

This contradicts the assumption that $\left\|u_{n}\right\|_{H^{1}} \rightarrow \infty$.

Remark 4.8. It is not in general clear whether the mountain-pass solutions we produce in Theorem 4.6 form a continuous family. Indeed, both the openness and closedness estimates along $\gamma$ depend on the stability condition $L>0$, which we expect to fail for the mountain-pass solutions. We can say more near $T_{0}$, as the continuous family $\gamma$ bifurcates there (one can use the implicit function theorem as in Uhlenbeck [Uhl83, p. 157]). It is unclear whether the bifurcated solutions for $t=T_{0}-\epsilon$ coincide with the mountain-pass solutions we construct.

\section{The Weil-Petersson pairing}

In this section, we use the uniqueness of the solution of $F(u(t), t)$ on the solution curve $\gamma$ near $\gamma(0)=(0,0)$ to define a functional on a subspace of the space of minimal Lagrangian immersions in $\mathbb{C H}^{2}$. This functional turns out to have positive definite second variation over Teichmüller space: a scalar multiple of the Weil-Petersson pairing of holomorphic cubic differentials. In the case of closed minimal surface in a class of quasi-Fuchsian manifolds, a similar functional is shown [GHW10] to be a potential function for the classical Weil-Petersson metric on Teichmüller space. This is 
also an analog of the fact that the second variation of the energy functional for harmonic maps between closed surfaces yields the Weil-Petersson metric on Teichmüller space [Wol89]. We recall that the Weil-Petersson pairing of holomorphic cubic differentials $q_{1} d z^{3}$ and $q_{2} d z^{3}$ is defined as

$$
\left\langle q_{1}, q_{2}\right\rangle_{W P}=\int_{\Sigma} \frac{q_{1} \bar{q}_{2}}{g_{\sigma}^{3}} d A_{\sigma} .
$$

Let $M L I(\Sigma)$ be the space of minimal Lagrangian immersions of (the covering of) a closed surface $\Sigma$ into $\mathbb{C} \mathbb{H}^{2}$ and $M L I_{\epsilon}(\Sigma)$ be the subspace of $M L I(\Sigma)$ such that the solution on $\gamma$ is unique $(\epsilon$ - close to $\gamma(0)=(0,0))$. We have the complex dimension of $M L I_{\epsilon}(\Sigma)$ is equal to $8 g-8$. Now the following functional is well-defined:

$$
\mathcal{A}: M L I_{\epsilon}(\Sigma) \rightarrow \mathbb{R}, \quad(\sigma, q) \mapsto-\int_{\Sigma} e^{u(t)} d A_{\sigma},
$$

i.e., it maps a minimal Lagrangian immersion to negative of the surface area associated to the metric $e^{u} g_{\sigma}$. We consider this family of functions $\mathcal{A}(t)=\mathcal{A}(\gamma(t))$ and its variations.

Theorem 5.1. We have the following

(i) $\mathcal{A}(0)=4 \pi(1-g)$;

(ii) $\dot{\mathcal{A}}=\left.\frac{d \mathcal{A}}{d t}\right|_{t=0}=0$;

(iii) $\ddot{\mathcal{A}}=\left.\frac{d^{2} \mathcal{A}}{d t^{2}}\right|_{t=0}=16 \int_{\Sigma}\|q\|^{2} d A_{\sigma}$.

Proof. (i) Since $\gamma(0)=(0,0)$, we have $\mathcal{A}(0)=-\int_{\Sigma} d A_{\sigma}=4 \pi(1-g)$.

(ii) We denote $\dot{u}=\left.\frac{d u}{d t}\right|_{t=0}$, and differentiate equation (3.1) with respect to $t$ to find:

$$
\Delta \dot{u}-32 t\|q\|^{2} e^{-2 u}+32 t^{2}\|q\|^{2} e^{-2 u} \dot{u}-2 e^{u} \dot{u}=0 .
$$

Now take value at $\gamma(0)$, we have

$$
(\Delta-2) \dot{u}=0,
$$

so $\dot{u}=0$ from the maximum principle. The claim (ii) now follows immediately.

(iii) We denote the operator $D=-2(\Delta-2)^{-1}$. This is a positive, self-adjoint (with respect to the $L^{2}$ inner product of functions $\left\langle f_{1}, f_{2}\right\rangle=\int_{\Sigma} f_{1} f_{2} d A_{\sigma}$ ) operator and $D(1)=1$. Note that this operator plays a fundamental role in the Weil-Petersson geometry of Teichmüller space [Wol86]. Now we differentiate equation (5.3) with respect to $t$ to find:

$$
\Delta \ddot{u}-32\|q\|^{2} e^{-2 u}+32 t^{2}\|q\|^{2} e^{-2 u} \ddot{u}-2 e^{u} \dot{u}^{2}-2 e^{u} \ddot{u}=0 .
$$

We evaluate above at $(0,0)$ to obtain

$$
(\Delta-2) \ddot{u}=32\|q\|^{2},
$$

and hence

$$
\ddot{u}=-16 D\left(\|q\|^{2}\right) .
$$

Now the claim (iii) follows from the self-adjointness of the operator $D$ and (5.5). 


\section{Acknowledgments}

The research of Huang is supported in part by a grant from CUNY-CSI Provost's scholarship and a CIRG-CUNY award, and he also has benefitted from helpful conversations at an AIM workshop "Dynamics of the Weil-Petersson geodesic flow"; the research of Loftin is supported in part by a Simons Collaboration Grant for Mathematicians 210124, and in part from from US National Science Foundation grants DMS 1107452, 1107263, 1107367 "RNMS: Geometric structures And Representation varieties" (the GEAR Network); and the research of Lucia is supported by projects MTM2008-06349-C03-01, MTM2011-27739-C04-01 (Spain) and 2009SGR345 (Catalunya), and a Simons Foundation Collaboration Grant for Mathematicians 210368 .

\section{References}

[AR73] A. Ambrosetti and P.H. Rabinowitz, Dual variational methods in critical points theory and applications, J. Funct. Anal. 14 (1973), 349-381.

[Bry87] R.L. Bryant, Minimal Lagrangian submanifolds of Kähler-Einstein manifolds, Differential geometry and differential equations (Shanghai, 1985), Lecture Notes in Mathematics, 1255, Springer, Berlin, 1987, pp. 1-12.

[FK80] H.M. Farkas and I. Kra, Riemann surfaces, Graduate Texts in Mathematics, 71, Springer-Verlag, New York, 1980.

[GHW10] R. Guo, Z. Huang and B. Wang, Quasi-Fuchsian three-manifolds and metrics on Teichmüller space, Asian J. Math. 14(2) (2010), 243-256.

[Gol99] W.M. Goldman, Complex hyperbolic geometry, Oxford Mathematical Monographs, The Clarendon Press Oxford University Press, New York, 1999, Oxford Science Publications.

[HL82] R. Harvey and H. Blaine Lawson, Jr., Calibrated geometries, Acta Math. 148 (1982), 47-157.

[HL12] Z. Huang and M. Lucia, Minimal immersions of closed surfaces in hyperbolic three-manifolds, Geom. Dedicata 158 (2012), 397-411, arXiv:1011.1313.

[Joy05] D. Joyce, Lectures on special Lagrangian geometry, Global theory of minimal surfaces, Clay Mathematics Proc. 2, 2005, pp. 667-695.

[Lab07] F. Labourie, Flat projective structures on surfaces and cubic holomorphic differentials, Pure Appl. Math. Q. 3(4) (2007), part 1, 1057-1099.

[LJ70] H.B. Lawson Jr., Complete minimal surfaces in $S^{3}$, Ann. Math. 92 (1970), 335-374.

[LM13] J. Loftin and I. McIntosh, Minimal Lagrangian surfaces in $\mathbb{C H}^{2}$ and representations of surface groups into $S U(2,1)$, Geom. Dedicata 162 (2013), 67-93.

[Lof01] J.C. Loftin, Affine spheres and convex $\mathbb{R P}^{n}$-manifolds, Amer. J. Math. 123(2) (2001), $255-274$.

[LYZ05] J. Loftin, S.-T. Yau, E. Zaslow, Affine manifolds, SYZ geometry and the "Y" vertex, J. Differ. Geom. 71(1) (2005), 129-158.

[MW93] M.J. Micallef and J.G. Wolfson, The second variation of area of minimal surfaces in fourmanifolds, Math. Ann. 295(2) (1993), 245-267.

[Oh90] Y.-G. Oh, Second variation and stabilities of minimal Lagrangian submanifolds in Kähler manifolds, Invent. Math. 101(2) (1990), 501-519.

[PP06] J.R. Parker and I.D. Platis, Open sets of maximal dimension in complex hyperbolic quasiFuchsian space, J. Differ. Geom. 73 (2006), 319-350.

[Sim68] J. Simons, Minimal varieties in riemannian manifolds, Ann. of Math. (2) 88 (1968), 62-105.

[Smo96] K. Smoczyk, A canonical way to deform a Lagrangian submanifold, arXiv:dg-ga/9605005v2, 1996.

[SW99] R. Schoen and J. Wolfson, Minimizing volume among Lagrangian submanifolds, Differential equations: La Pietra 1996 (Florence), Proc. Symp. Pure Mathematics, 65, Amer. Math. Soc., Providence, RI, 1999, pp. 181-199.

[SW01] R. Schoen and J. Wolfson, Minimizing area among Lagrangian surfaces: the mapping problem, J. Differ. Geom. 58(1) (2001), 1-86. 
[SYZ96] A. Strominger, S.-T. Yau and E. Zaslow, Mirror symmetry is T-duality, Nucl. Phys. B 479(1-2) (1996), 243-259.

[Uhl83] K.K. Uhlenbeck, Closed minimal surfaces in hyperbolic 3-manifolds, Seminar on minimal submanifolds, Ann. Math. Stud. 103, Princeton University Press, Princeton, NJ, 1983, pp. 147-168.

[Wan08] M.T. Wang, Some recent developments in Lagrangian mean curvature flows, Surveys in differential geometry. Vol. XII. Geometric flows, Surv. Differ. Geom., vol. 12, Int. Press, Somerville, MA, 2008, pp. 333-347.

[Wol86] S.A. Wolpert, Chern forms and the Riemann tensor for the moduli space of curves, Invent. Math. 85(1) (1986), 119-145.

[Wol89] M. Wolf, Teichmüller theory of harmonic maps, J. Differ. Geom. 29(2) (1989), 449-479.

Department of Mathematics, The City University of New York, Staten Island, NY 10314, USA

E-mail address: zheng.huang@csi.cuny.edu

Department of Mathematics and Computer Science, Rutgers University, Newark, Newark, NJ 07102, USA

E-mail address: loftin@rutgers.edu

Department of Mathematics, The City University of New York, Staten Island, NY 10314, USA

E-mail address: mlucia@math.csi.cuny.edu 